\title{
Near-Earth Object Exploration Leading to Human Visits
}

\author{
Daniel R. Adamo \\ 503-585-0025 \\ Independent Astrodynamics Consultant \\ adamod@earthlink.net
}

Paul A. Abell, NASA-Johnson Space Center

Robert C. Anderson, Jet Propulsion Laboratory/California Institute of Technology

Brent W. Barbee, NASA-Goddard Space Flight Center

Thomas D. Jones, Senior Research Scientist, Institute for Human and Machine Cognition

Daniel D. Mazanek, NASA Langley Research Center

Gregg Podnar, Robot Systems Architect, Aeolus Robotics 


\section{Near-Earth Object Exploration Leading to Human Visits}

\section{Introduction}

The Global Exploration Roadmap, promoted by NASA and 13 other space agencies through the International Space Exploration Coordination Group, currently proceeds from humans at lunar destinations directly to humans at Mars [1, p. 3]. Following this path entails spanning a formidable leap in human space flight (HSF) capability. Roundtrip mission duration $\Delta t$ jumps from days or weeks for the Moon to years for Mars. Two-way communications with Earth have a light-time lag of 2.4 seconds at the Moon, and reasonably routine conversation is possible. Depending on positions of Earth and Mars about the Sun, two-way communications between these planets have light-time delays from 6.3 to 44 minutes. Thus, levels of HSF systems reliability and crew autonomy required at the Moon must be multiplied many times to reach Mars, conduct productive exploration operations there, and return to Earth safely.

Bridging the HSF capability gap between the Moon and Mars are "steppingstone" destinations provided by near-Earth objects (NEOs). Mission analysis demonstrates many NEOs are intermediate in accessibility between the Moon and Mars as quantified by fundamental roundtrip change-in-velocity $\Delta v$ and $\Delta t$ metrics (see Figure 1). According to NASA's Center for Near-

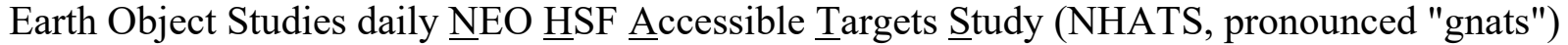
processing, the subset of known NEOs more accessible to HSF than Mars currently numbers 3046 [2]. With NEO steppingstone destinations, HSF can incrementally increase $\Delta v$ and $\Delta t$ until Mars visits are relatively routine. Furthermore, NEOs are compelling destinations in their own right because they offer the opportunity to investigate more exploration themes than any other HSF destination [3]:

1) Planetary science on bodies relatively unmodified since early Solar System formation,

2) Planetary defense on bodies having potential to impact Earth with disastrous effects, and

3) In-situ resource utilization (ISRU) on bodies with materials of strategic and/or commercial value transportable throughout the inner Solar System.

As has been the case since the Apollo Program in the 1960s, robotic precursor missions help pave the way to an exploration destination such as the Moon by retiring risk for subsequent HSF visits $[4,5]$. In connection with the current Artemis Program's human return to the Moon, robotic precursors are being instigated through NASA's Commercial Lunar Payload Services initiative [6]. These lunar precursors tend to be science-centered, but they also incorporate technology demonstration objectives addressing HSF and ISRU knowledge gaps.

Other HSF knowledge gaps exist at NEO destinations, but Earth-based observations, together with robotic precursors such as JAXA's Hayabusa2 [7] and NASA's OSIRIS-REx [8], are already beginning to fill them. When observations and precursor missions described in this paper lead to safe and rewarding NEO visits by humans, exploration productivity at these destinations will be increased by orders of magnitude, as was demonstrated during Apollo lunar surface operations [9]. Aided by modern technology, a synergistic collaboration between humans and robots at NEOs promises to dwarf any off-Earth exploration productivity previously demonstrated. Future robotic visits to NEOs should plan to lay the groundwork for this collaboration. 


\section{Near-Earth Object Exploration Leading to Human Visits}

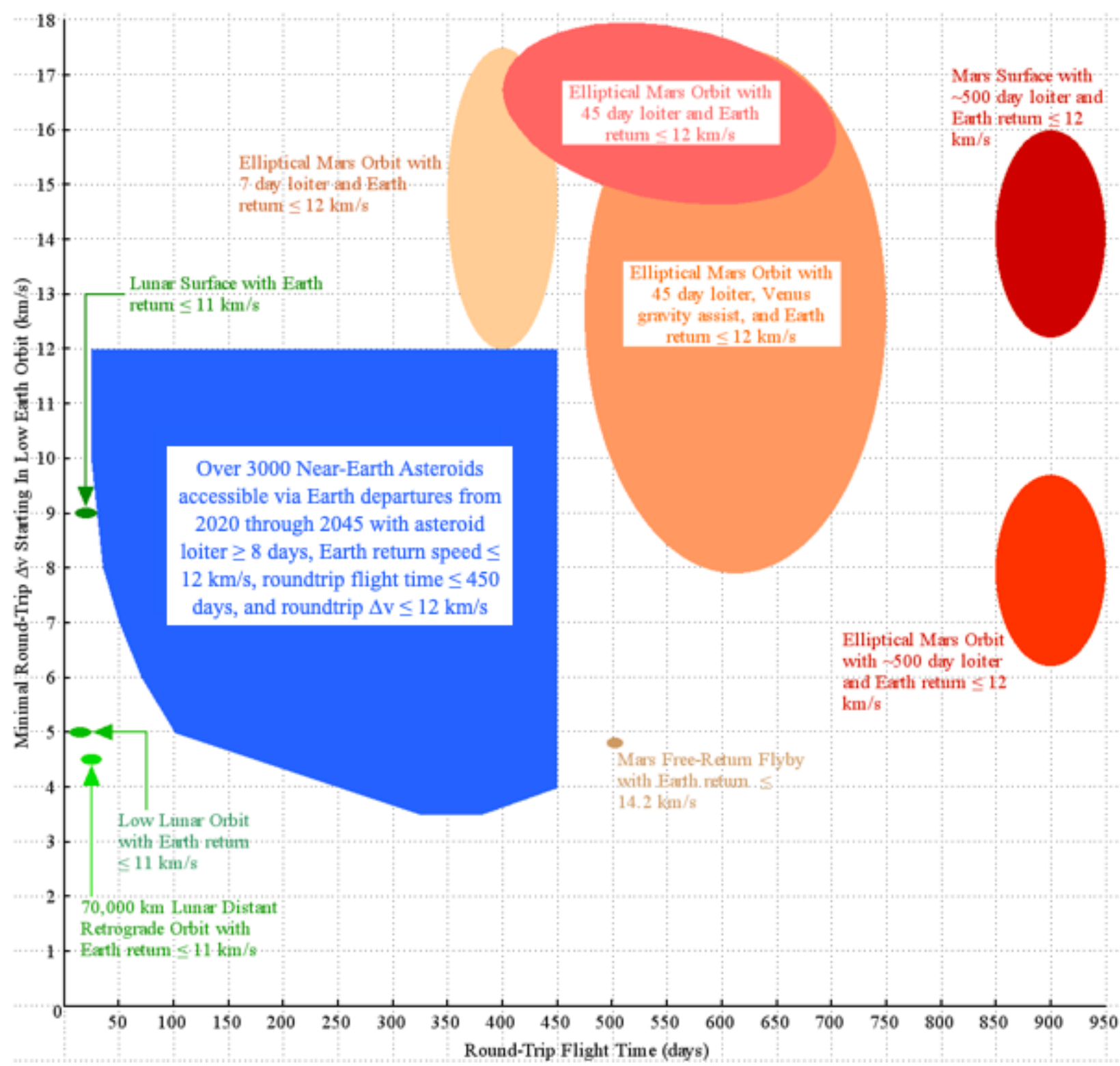

Figure 1. Fundamental metrics $\Delta v$ (vertical axis) and $\Delta t$ (horizontal axis) define colored regions associated with destinations being targeted for HSF in future decades. Green regions are minimal $\Delta t$ roundtrips to the Moon, red/orange regions are Mars roundtrips, and intermediate "steppingstone" NEO roundtrips fall into the blue region. Because direct entry is assumed on Earth return, each region is annotated with maximum geocentric speed at $+\mathbf{1 2 2}$ kilometers altitude as controlled by $\Delta v$. This graphic is adapted from [10].

\section{Institutional Support to NEO Exploration}

Surveys to detect, catalog, and characterize NEOs with remote sensing techniques are justified primarily by planetary defense objectives such as the George E. Brown NEO Survey [11, p. xi]. This Congressionally mandated survey will not be completed before its 2020 deadline.

Nonetheless, NASA's NEO Surveillance Mission (NEOSM), first funded in 2019, is a means of significantly contributing to the survey's other goals after a launch planned no earlier than 2025 


\section{Near-Earth Object Exploration Leading to Human Visits}

[12]. Unofficial estimates forecast $90 \%$ of NEOs larger than 140 meters in diameter might be cataloged after a decade of NEOSM operations.

As noted in Section 1's Introduction, NEOs more accessible to HSF than Mars are abundant. Nevertheless, many of these NHATS-compliant destinations are considered to be of insufficient size to justify costs and risks of a human visit. The minimal acceptable NEO size for human exploration has yet to be established, but these small and other marginally observable NEOs are typically discovered during close encounters with Earth and all NEO surveillance sensors. Postencounter, NEOs are often lost to terrestrial sensors before an accurate orbit can be determined to support reliable HSF mission planning [13]. Still other NEOs are in orbits very similar to Earth's, and they spend decades on the other side of the Solar System before returning to Earth's vicinity and NHATS-compliant accessibility levels [14, Figure 11]. Consequently, remote sensing is necessary to identify the greatest possible number of NEO destinations for practical HSF missions, and only a few of these missions can be flown at any particular time.

In addition to highly precise orbit determination, other NEO remote-sensing capabilities are provided by Solar System radar facilities at Goldstone, California and Arecibo, Puerto Rico. For sufficiently close Earth approaches, radar can characterize important NEO parameters including size/shape, topography, spin state, and the presence of sufficiently detectable companion objects. Such data can help identify appealing HSF destinations or exclude others due to unacceptable hazards [3, Table 3.1]. Radar facilities require ongoing funds for staffing and facilities maintenance, as recognized by NASA's Small Bodies Assessment Group (SBAG) $\left[15,8^{\text {th }}\right.$ finding from SBAG 19].

The probability that a sufficiently large, NHATS-compliant NEO destination will be accessible at a programmatically desirable HSF launch date may be remote. Therefore, additional NEO discoveries, orbit refinements, and physical characteristics provided by Earth-based sensors and an operational NEOSM are welcomed by planetary defense stakeholders, together with HSF and robotic mission planners. Survey missions like NEOSM have been advocated by SBAG since $2011\left[15,1^{\text {st }}\right.$ finding from SBAG 5], and this support is expected to continue, motivated in part by SBAG's association with HSF [3, Goal 3].

Because copious sample mass will undoubtedly be returned by HSF expeditions to off-Earth destinations, state-of-the-art curation facilities on Earth are an inescapable requirement.

Curation is necessary to provide planetary protection for Earth's biosphere, sample preservation, and sample distribution to qualified researchers over time. Sample returns by Hayabusa2 in 2020 [7] and OSIRIS-REx in 2023 [8] illustrate the time-critical need for adequate curation facilities and personnel. Sufficient financial support for world-class sample curation is an investment as necessary to the study of NEOs as are the funds required to collect and return samples to Earth [15, $3^{\text {rd }}$ finding from SBAG 22].

\section{High-Latency Robotic Precursors at NEOs}

High-latency robotic precursors are deployed at destinations considerably more distant from their operators than the Moon is from Earth. Under high-latency conditions, an operator's command to the robotic precursor cannot be confirmed by the operator before tens of seconds to days have elapsed [17, p. 101 Latency definition]. This is the mode under which all NEO exploration 


\section{Near-Earth Object Exploration Leading to Human Visits}

missions \{NEAR Shoemaker at (433) Eros, Hayabusa at (25143) Itokawa, Hayabusa2 at (162173) Ryugu, and OSIRIS REx at (101955) Bennu\} have heretofore operated.

Robotic autonomy required to address high latency at NEO destinations can be challenging, particularly when performing proximal "touch and go" operations necessary to obtain samples. For example, the Hayabusa spacecraft suffered uncontrolled contact with (25143) Itokawa during one of its sampling attempts [17]. The first sampling attempt by OSIRIS-REx at (101955) Bennu has recently incurred two months' postponement due in part to ensuring safe operations near an unexpectedly rugged surface during planned autonomous sampling [18, May 20, 2020 report].

Nevertheless, high-latency robotic precursors have demonstrated sample return capability at NEOs [17], along with detailed surface mapping [18, March 6, 2020 report], safe proximity operations [18, March 4, 2020 report], proximal debris identification [18, December 14, 2019 report], and ISRU prospecting [18, December 10, 2018 report]. All of these capabilities are relevant to discovering environmental properties Earth-based sensing has missed, identifying the most compelling HSF destinations among NEO candidates, and reducing risks these candidates pose to safe, productive operations before humans even depart for such destinations.

Unlike HSF missions, robotic precursors are normally not under minimal $\Delta t$ constraints. Furthermore, robotic missions do not need to make a roundtrip from Earth at all if NEO sample return is not required. A one-way mission profile, coupled with much lower precursor mass than HSF transports, permit the acceptable $\Delta v$ to be increased or the cost of launch to be reduced. Thus, multi-year outbound transits allow precursor missions to access virtually any NHATScompliant NEO following launch during any programmatically desirable year. It is then possible to plan precursor arrival one or more years before a crew would depart Earth for that NEO destination on a less prolific mission opportunity with lower $\Delta t$ and $\Delta v$ constraints. Using this dual-mission campaign strategy, along with sufficiently flexible HSF capabilities and training, precursor findings can conclusively determine whether or not costs and risks for HSF to the prospective NEO destination are justified before committing a crew to launch.

\section{Low-Latency Robotic Surrogates at NEOs}

Low-latency robotic surrogates are deployed at destinations considerably less distant from their operators than the Moon is from Earth. When an operator is closer than 30,000 kilometers to a robotic surrogate, a dedicated communications link will have roundtrip light-time latency less than 200 milliseconds. Aided by state-of-the-art robotic interfaces such as high-resolution zoom optics, multispectral binocular vision, precision manipulators, and haptic feedback, the operator enjoys an immersive cognitive experience termed low-latency telepresence (LLT) at the asset's location. During LLT exploration, any delay in a command/response action becomes transparent to the operator, even while highly dexterous tasks are performed [16, p. 101 Latency definition].

One of the major benefits from LLT is the operator explores from a shirtsleeve habitat environment, free from hazards and timeline overhead associated with extra-vehicular activity (EVA) [16, Section 5.1.1]. Every hour of EVA requires about two hours of crew activity, typically involving additional crewmembers in support of those who are to work outside a pressurized habitat. Activities include retrieving/checking/donning/doffing/restowing 


\section{Near-Earth Object Exploration Leading to Human Visits}

equipment, pure oxygen pre-breathe, airlock depressurization/re-pressurization, and recharging EVA consumables. Because of this overhead, there is no instance of back-to-back, multi-shift EVAs in the history of HSF. In addition, EVA duration is consumables-limited by the mass and bulk a human can reasonably carry. The longest U.S. EVA duration on record is less than 9 hours [19].

With LLT, continuous, round-the-clock exploration operations could be practical if crew size and habitat design permit it. Indeed, such operations may be advisable for some NEO visits because crew time at the destination might be limited to approximately 10 days by mission $\Delta v$ and $\Delta t$ constraints. Regardless of whether or not multi-shift operations are conducted, productivity levels associated with LLT exploration at NEOs would greatly exceed those achieved during high-latency robotic missions, and even Apollo lunar surface sorties, due to greater operational efficiencies [16, p. 87].

Using LLT, an operator is insulated from hazardous EVA activities such as clambering over unstable NEO terrain in microgravity [16, Section 8.3]. A more expendable and durable robotic surrogate incurs these risks and can be designed for specialized tasks, such as anchoring in regolith, far better than an astronaut in pressurized EVA garb. This greater risk tolerance leads to synergistic exploration capabilities for environments no in-situ human or autonomous robot would otherwise choose to access. For example, consider negotiating the debris field associated with expelled material about an active NEO. Neither a space-suited human (susceptible to contamination hazards from debris contact) nor an autonomous robot (limited in collision management capabilities) would enter such an environment without incurring considerable risk. But a well-equipped robotic surrogate, under human control via immersive LLT interfaces, could reliably fly sorties through the debris with precision thrusters and manipulators to gently push aside, examine, or sample debris in its path.

When NEO samples are collected by an LLT robotic surrogate, as opposed to a human during EVA, two important benefits accrue. First, Earthly contamination of the sample is isolated to the surrogate and sample container, whose cleanliness can be established and maintained far more rigorously than EVA equipment worn or handled by humans. Second, all humans visiting the NEO can be isolated from potentially toxic substances in the sample contaminating EVA equipment that must subsequently be worn or handled.

Experiments evaluating LLT tasks with Earthbound robotic surrogates have already been conducted by International Space Station crewmembers [16, Section 7.1.1]. Prior to deploying assets at NEOs, LLT systems and techniques would benefit from further development during lunar surface exploration [16, Sections 8.1 and 8.2]. Exploration of NEOs with LLT can itself feed forward to exploration of Mars. An example would be humans in a subsurface Deimos habitat controlling robotic surrogates on the surface of Mars [16, Figure 7.4].

Robotic LLT surrogates will undoubtedly be on the essential equipment stowage manifest for HSF missions to NEOs if for no other purpose than to provide mission-critical redundancy with hardware pre-positioned at those destinations. Surrogates should have high functional commonality with robotic systems dispatched to the same destinations on precursor missions. Precursor systems should ideally be capable of operating over a range of autonomy modes to be 


\section{Near-Earth Object Exploration Leading to Human Visits}

compatible with data latencies measured in days down to those of LLT. If such a precursor confirms a candidate NEO is indeed a compelling HSF destination, it could double as an LLT surrogate when humans arrive. Less productive LLT exploration with the precursor could also be conducted shortly before crew arrival at the NEO and shortly after departure from it, further contributing to planetary science, planetary defense, and ISRU data return from this destination $[16$, Section 5.1.2].

\section{Conclusion}

Highly accessible to HSF, NEOs are strategic destinations in any informed and prudent exploration strategy leading from the Moon to Mars. Because they combine exploration themes relating to planetary science, planetary defense, and ISRU, NEOs are also among the most compelling off-Earth destinations in their own right.

High-latency robotic missions to NEOs have already proven their considerable value as HSF precursors. When humans follow to the most worthwhile NEO destinations, precursor systems have nearly complete feed-forward applications, even to the point of being repurposed as LLT surrogates for visiting crews. With LLT, human exploration at NEO destinations is freed from the risks, limitations, and timeline overhead posed by EVAs. Thus, time spent by humans actually exploring proximal to NEOs is maximized by LLT, while productivity during that time is greatly increased with respect to exploration in EVA mode.

With these precepts in mind, two-mission exploration campaigns are highly recommended for NEO destinations. A candidate HSF destination NEO is first identified by remote sensing. A necessary requirement for any such destination is at least one HSF mission opportunity meeting highly constrained $\Delta t$ and $\Delta v$ limits and having Earth departure sufficiently far in the future. A high-latency robotic precursor mission is then sent to the candidate NEO, arriving at least a year before the HSF mission opportunity would depart Earth. This mission will, if past NEO exploration by NASA and JAXA is any precedent, return impressive rewards in planetary science, planetary defense, and ISRU data. If findings from these data justify associated costs and risks, the campaign's HSF mission is launched and conducts LLT exploration at the NEO.

The highly productive nature of LLT will greatly expand knowledge of any NEO visited by humans and cooperative robotic surrogates using this exploration mode. These visits will also ensure a safe, sustainable, and incremental path on the HSF exploration roadmap to Mars.

\section{References}

[1] International Space Exploration Coordination Group, The Global Exploration Roadmap, NASA-HQ, NP-2018-01-2502-HQ, G-327035, 2018. ${ }^{1}$

[2] https://cneos.jpl.nasa.gov/nhats/ (accessed "Data Table" with "Use Unconstrained Settings" on 19 May 2020).

\footnotetext{
${ }^{1}$ This publication may be downloaded at https://www.nasa.gov/sites/default/files/atoms/files/ger_2018_small_mobile.pdf (accessed 19 May 2020).
} 


\section{Near-Earth Object Exploration Leading to Human Visits}

[3] Small Bodies Assessment Group (SBAG), Goals and Objectives for the Exploration and Investigation of the Solar System's Small Bodies, $2020{ }^{2}$

[4] https://nssdc.gsfc.nasa.gov/planetary/lunar/lunarorb.html (accessed 19 May 2020).

[5] https://nssdc.gsfc.nasa.gov/planetary/lunar/surveyor.html (accessed 19 May 2020).

[6] https://www.nasa.gov/content/commercial-lunar-payload-services-overview (accessed 19 May 2020).

[7] https://solarsystem.nasa.gov/missions/hayabusa-2/in-depth/ (accessed 19 May 2020).

[8] https://www.nasa.gov/osiris-rex (accessed 19 May 2020).

[9] https://www.hq.nasa.gov/alsj/main.html (accessed 20 May 2020).

[10] https://www.lpi.usra.edu/sbag/science/NHATS_Accessible_NEAs_Summary.png (accessed 19 May 2020).

[11] NRC Committee to Review Near-Earth Object Surveys and Hazard Mitigation Strategies, Defending Planet Earth: Near-Earth Object Surveys and Hazard Mitigation Strategies:

Final Report, National Academies Press, 2010. ${ }^{3}$

[12] https://spacenews.com/nasa-to-develop-mission-to-search-for-near-earth-asteroids/ (accessed 20 May 2020).

[13] https://cneos.jpl.nasa.gov/news/news204.html (accessed 20 May 2020).

[14] D. R. Adamo, "Initial Near-Earth Object Accessibility Insights from the 'NHATSchecker' Utility", AAS 19-623, Advances in the Astronautical Sciences, Vol. 171, pp. 2885-2904, Univelt, 2020. ${ }^{4}$

[15] https://www.lpi.usra.edu/sbag/ (accessed 21 May 2020).

[16] R. C. Anderson et al, Space Science Opportunities Augmented by Exploration Telepresence, Keck Institute for Space Studies, 2020. ${ }^{5}$

[17] https://curator.jsc.nasa.gov/hayabusa/ (accessed 21 May 2020).

[18] https://www.asteroidmission.org/latest-news/ (accessed 21 May 2020).

[19] https://www.nasa.gov/mission_pages/shuttle/shuttlemissions/archives/sts-102.html (accessed 24 May 2020).

\footnotetext{
2 This document may be downloaded at https://www.lpi.usra.edu/sbag/goals/SBAG_Goals_Document_2020.pdf (accessed 19 May 2020).

3 This document may be downloaded at http://www.nap.edu/catalog/12842.html (accessed 20 May 2020).

${ }^{4}$ A preprint of this paper may be downloaded at http://www.aiaahouston.org/adamo_astrodynamics/ as an email attachment to the "ATIG_106" archive (accessed 20 May 2020).

5 This document may be downloaded from https://www.kiss.caltech.edu/final_reports/Telepresence_Final_Report.pdf (accessed 8 June 2020).
} 